\title{
FATOR CAPACIDADE DE FÓSFORO EM SOLOS DE PERNAMBUCO MINERALOGICAMENTE DIFERENTES E INFLUÊNCIA DO pH NA CAPACIDADE MÁXIMA DE ADSORÇÃO'
}

\author{
Phosphate capacity factor in mineralogically different soils in Pernambuco and \\ the influence of $\mathbf{p H}$ on the maximum capacity of adsorption ${ }^{1}$ \\ Fábio Broggi ${ }^{2}$, Alexandre Campelo de Oliveira ${ }^{3}$, Fernando José Freire ${ }^{4}$, \\ Maria Betânia Galvão dos Santos Freire ${ }^{3}$, Clístenes Williams Araújo do Nascimento ${ }^{3}$
}

\begin{abstract}
RESUMO
O Fator Capacidade de Fósforo (FCP) é definido pela razão de equilíbrio entre o fator quantidade de $\mathrm{P}(\mathrm{Q})$ e o fator intensidade (I) e representa uma medida da capacidade do solo em manter um determinado nível de P em solução. As características e o teor dos constituintes minerais da fração argila são responsáveis por uma maior ou menor FCP, interferindo nas relações soloplanta. Por outro lado, o pH do solo tem, em alguns casos, mostrado-se com efeito na adsorção e, em outros, com pequena e não consistente alteração na Capacidade Máxima de Adsorção de P (CMAP). Objetivou-se, neste trabalho, determinar o FCP de solos mineralogicamente diferentes em Pernambuco; correlacionar características físicas e químicas dos solos com o FCP; e avaliar o efeito do $\mathrm{pH}$ na CMAP. Amostras subsuperficiais de quatro solos, mineralogicamente diferentes, foram caracterizadas química e fisicamente e determinado o FCP. Essas amostras foram corrigidas com $\mathrm{CaCO}_{3}$ e $\mathrm{MgCO}_{3}$ na proporção 4:1 e incubadas por 30 dias, com exceção do Vertissolo. Determinou-se a CMAP antes e após a correção dos solos. O experimento consistiu de um fatorial 4 x 2 (quatro solos com e sem correção), distribuídos em blocos ao acaso, com três repetições. As características dos solos que melhor refletiram o FCP foram o P remanescente (P-rem) e a CMAP. Independentemente dos constituintes mineralógicos da fração argila, solos com elevados teores de alumínio apresentaram aumento da CMAP com a correção. A energia de adsorção (EA) nos solos corrigidos foi, em média, significativamente menor, independentemente do solo.
\end{abstract}

Termos para indexação: Adsorção de fósforo, poder tampão de fosfato, CMAP.

\begin{abstract}
Phosphate Maximum Capacity (FCP) is defined by the ratio of equilibrium between the amount of factor P (Q) and factor intensity (I) and represents a measure of the soil ability to maintain a certain level of $\mathrm{P}$ in solution. The characteristics and content of the constituents of clay minerals are responsible for a greater or lesser FCP, interfering in soil-plant relations. Moreover, the soil $\mathrm{pH}$ has affected adsorption, and in other cases, it has shown small and inconsistent change in the maximum adsorption capacity of $\mathrm{P}$ (CMAP). Thus, this study aimed to determine the different FCP soil mineralogy in Pernambuco; to correlate physical and chemical characteristics of soils with PBC and to evaluate the effect of $\mathrm{pH}$ on the CMAP. Subsurface soil samples from four different soils were characterized chemically and physically determined, and the $\mathrm{PBC}$ was determined. These samples were corrected with $\mathrm{CaCO}_{3}$ and $\mathrm{MgCO}_{3}$ in a 4:1 ratio and incubated for 30 days, except the Vertisol. The CMAP was determined before and after correction of the soil. The experiment consisted of a 4 x 2 factorial (four soils with and without correction), distributed in randomized blocks with three replicates. Soil characteristics that best reflected the PBC were the remaining P (P-rem) and MPAC. Regardless of the constituents of clay mineralogy, soil with high aluminum levels had increased CMAP after correction. The energy of adsorption (EA) in the limed soils was on average significantly lower, regardless of the soil.
\end{abstract}

Index terms: Phosphorus adsorption, phosphate buffer power, CMAP.

(Recebido em 16 de junho de 2009 e aprovado em 28 de julho de 2010)

\section{INTRODUÇÃO}

Os minerais coloidais encontrados na fração argila são responsáveis por importantes reações físicas e químicas dos solos. O processo de adsorção de íons da solução do solo é um exemplo desse tipo de fenômeno. Nesse contexto, o P tem sido objeto de um grande número de trabalhos, por ser o nutriente que mais onera os custos de produção agrícola, pela baixa relação matéria-seca produzida/kg de nutriente aplicado. Isso se deve tanto à sua deficiência natural, principalmente em solos tropicais (Silva et al., 2003; Carneiro et al., 2008), quanto à sua indisponibilidade em solos argilosos e intemperizados (Rolim et al., 2004).

\footnotetext{
${ }_{1}^{1}$ Parte da Dissertação do primeiro autor, apresentada à Universidade Federal Rural de Pernambuco/UFRPE, para obtenção do título de Mestre ETH Bioenergia - Alto Taquari, MT

${ }^{3}$ Universidade Federal Rural de Pernambuco/UFRPE - Departamento de Agronomia - Recife, PE

${ }^{4}$ Universidade Federal Rural de Pernambuco/UFRPE - Departamento de Agronomia - Rua Dom Manoel de Medeiros - s/n - Dois Irmãos - 52171-900 Recife, PE - f.freire@depa.ufrpe.br
} 
O fator capacidade de fósforo (FCP) ou poder tampão de fosfato é definido pela razão de equilíbrio entre a quantidade de $\mathrm{P}(\mathrm{Q})$ e o P em solução (I) e representa uma medida da capacidade do solo em manter um nível determinado de $\mathrm{P}$ em solução. As características e o teor dos constituintes minerais da fração argila são responsáveis pela velocidade do processo de passagem do $\mathrm{P}$ lábil para o $\mathrm{P}$ não-lábil (Gonçalves et al., 1989).

Fox \& Searle (1978) indicaram que o processo de adsorção pode acontecer preferencialmente, segundo uma ordem de predomínio dos seguintes minerais: argilas $2: 1<$ argilas $1: 1<$ óxidos cristalinos de $\mathrm{Fe}$ e $\mathrm{Al}<$ óxidos amorfos de Fe e Al. A adsorção de fosfato pelos solos é influenciada pelos minerais que apresentam grupamentos superficiais $\mathrm{Fe}-\mathrm{OH}$ e $\mathrm{Al}-\mathrm{OH}$, nos quais o fosfato pode ser adsorvido por meio de troca de ligantes (quimiossorção) com o estabelecimento de ligações covalentes, além disso, sua forma estrutural e sua superfície específica potencializam o efeito da adsorção (Mesquita Filho \& Torrent, 1993).

Componente importante no processo de adsorção é a reação do solo (Tirlone et al., 2009). Com o aumento do $\mathrm{pH}$, a carga superficial de partículas do solo torna-se mais negativa, diminuindo a adsorção entre fosfato e a superfície adsorvente (Posner \& Bowden, 1980; Haynes, 1984; Barrow, 1985). Entretanto, Vasconcelos et al. (1974), observaram pequena e não consistente alteração na CMAP em Latossolos do Cerrado brasileiro. A presença de cátions trocáveis como $\mathrm{Al}^{3+}, \mathrm{Ca}^{2+}, \mathrm{K}^{+}$e $\mathrm{Na}^{+}$, também afetam esse relacionamento da CMAP com o pH (Haynes, 1984).

Realizou-se, este trabalho, para determinar o FCP de solos mineralogicamente diferentes; correlacionar características físicas e químicas dos solos com o FCP; e avaliar o efeito do $\mathrm{pH}$ na CMAP.

\section{MATERIAL E MÉTODOS}

As amostras de solos foram coletadas em subsuperfície em diferentes regiões de Pernambuco
(Tabela 1), secas ao ar, destorroadas e passadas em peneira com malha de $2 \mathrm{~mm}$, tanto para a instalação do experimento, como para as análises físicas e químicas, que foram realizadas conforme Empresa Brasileira de Pesquisa Agropecuária Embrapa (1997) (Tabela 2). Os solos foram selecionados tomando-se como referência, tanto o teor, como a constituição mineralógica da fração argila (Figura 1).

$\mathrm{O} P$ remanescente (P-rem) foi determinado de acordo com Alvarez et al. (2000). Para avaliar a CMAP, amostras de 2,5 $\mathrm{cm}^{3}$ de Terra Fina Seca ao Ar (TFSA) receberam 11 doses de $\mathrm{P}\left(\mathrm{KH}_{2} \mathrm{PO}_{4}\right.$ p.a. $)$ no intervalo de 0 a $110 \mathrm{mg} \mathrm{L}^{-1}$ para o $\mathrm{LAd}_{1}$; 0 a $260 \mathrm{mg} \mathrm{L}^{-1}$ para o LAd, LVAd, e NVdf e de 0 a $80 \mathrm{mg} \mathrm{L}^{-1}$ para o VEo (Alvarez et al., 2000). As doses foram aplicadas utilizando-se $25 \mathrm{~mL}$ de uma solução de equilíbrio $\left(\mathrm{CaCl}_{2} 2 \mathrm{H}_{2} \mathrm{O} 0,01 \mathrm{~mol} \mathrm{~L}^{-1}\right.$, contendo as diferentes doses de P). Agitou-se em enlenmeyer de $125 \mathrm{~mL}$ por 24 horas, centrifugou-se por 5 minutos a $3.000 \mathrm{rpm}$ e filtrou-se, determinando-se a concentração de $\mathrm{P}$ no sobrenadante por colorimetria (Braga \& Defelipo, 1974). Utilizou-se a isoterma de Langmuir linearizada $(\mathrm{C} / \mathrm{q}=1 / \mathrm{ab}+1 / \mathrm{bC})$ para determinar a CMAP $(\mathrm{b})$, expressa em $\mathrm{mg} \mathrm{cm}^{-3}$; e a constante (a), relacionada com a energia de adsorção (EA), expressa em $\mathrm{L} \mathrm{mg}^{-1}$.

Realizou-se a separação da fração argila das amostras de solo para preparação das lâminas para análise mineralógica por difratometria de raios-X (Jackson, 1969). As leituras das amostras foram realizadas em difratômetro equipado com tubo de cobre, utilizando-se radiação $\mathrm{CuK} \alpha$ operado a $20 \mathrm{~mA}$ e $40 \mathrm{kV}$.

Utilizou-se o método proposto por Coffin (1963) e Schwertmann \& Taylor (1989) para a avaliação do grau de cristalinidade dos óxidos de ferro presentes na fração argila, sendo os teores de $\mathrm{Fe}$ amorfo $\left(\mathrm{Fe}_{\text {oxalato }}\right)$ e Fe cristalino $\left(\mathrm{Fe}_{\text {ditionito }}\right)$ determinados por espectrofotometria de absorção atômica.

Para correção da acidez, a exceção do VEo (pH 7,0), os solos foram incubados com uma mistura de $\mathrm{CaCO}_{3} \mathrm{e}$ $\mathrm{MgCO}_{3}$, numa relação molar de $4: 1$, por um período de 60 dias e a quantidade de corretivo estimada pela acidez potencial ( $\mathrm{H}+\mathrm{Al})$ dos solos (Kaminski et al., 2002).

Tabela 1 - Identificação, classe, horizonte, profundidade e procedência das amostras de solo, nas diferentes regiões do Estado de Pernambuco.

\begin{tabular}{lccc}
\hline \multicolumn{1}{c}{ Identificação/Classe } & Horizonte & Profundidade & Procedência \\
\hline $\mathrm{LAd}_{1}$ - Latossolo Amarelo distrófico & $\mathrm{Bw}_{3}$ & $132-155+\mathrm{cm}$ & Igarassú/PE $^{(1)}$ \\
$\mathrm{LAd}_{2}$ - Latossolo Amarelo distrófico & $\mathrm{Bw}_{1}$ & $74-155 \mathrm{~cm}$ & ${\text { Tamandaré} / \mathrm{PE}^{(2)}}^{(2)}$ \\
$\mathrm{NVdf}$ - Nitossolo Vermelho distroférrico & $\mathrm{B}_{\text {nítico }}$ & $30-56 \mathrm{~cm}$ & ${\mathrm{Cabo} / \mathrm{PE}^{(2)}}^{(3)}$ \\
VEo - Vertissolo Ebânico órtico & $\mathrm{CV}$ & $5-25 \mathrm{~cm}$ & Cachoeirinha/PE $^{(3)}$ \\
\hline
\end{tabular}

${ }^{(1)}$ Zona da Mata Norte; ${ }^{(2)}$ Zona da Mata Sul; ${ }^{(3)}$ Agreste Meridional. 
Tabela 2 - Caracterização física e química das amostras de solo.

\begin{tabular}{|c|c|c|c|c|}
\hline \multirow{2}{*}{ Característica } & \multicolumn{4}{|c|}{ Solo } \\
\hline & $\mathrm{LAd}_{1}$ & $\mathrm{LAd}_{2}$ & NVdf & VEo \\
\hline Areia $\left(\right.$ dag kg $\left.{ }^{-1}\right)$ & 76,8 & 24,2 & 11,8 & 29,8 \\
\hline Silte $\left(\right.$ dag kg $\left.{ }^{-1}\right)$ & 4,0 & 3,2 & 17,5 & 15,5 \\
\hline Argila (dag kg $\left.{ }^{-1}\right)$ & 19,2 & 72,6 & 70,7 & 54,7 \\
\hline Ds $\left(\mathrm{g} \mathrm{cm}^{-3}\right)^{(1)}$ & 1,57 & 1,22 & 1,12 & 1,72 \\
\hline $\operatorname{Dp}\left(\mathrm{g} \mathrm{cm}^{-3}\right)^{(2)}$ & 2,63 & 2,77 & 2,89 & 2,56 \\
\hline Cond. hidráulica $\left(\mathrm{cm} \mathrm{h}^{-1}\right)$ & 10,18 & 6,04 & 5,81 & 0,38 \\
\hline $\operatorname{Cc}(\%)^{(3)}$ & 7,94 & 29,51 & 39,7 & 39,53 \\
\hline $\operatorname{PMP}(\%)^{(4)}$ & 4,67 & 21,95 & 29,6 & 27,48 \\
\hline $\mathrm{pH} \mathrm{H} \mathrm{H}_{2} \mathrm{O}(1: 2,5)$ & 4,5 & 4,7 & 4,4 & 6,8 \\
\hline $\mathrm{pH} \mathrm{KCl}$ & 3,9 & 4,5 & 4,1 & 5,2 \\
\hline P Mehlich-1 (mg dm $\left.{ }^{-3}\right)$ & 0,35 & 0,0 & 2,36 & 495,6 \\
\hline $\mathrm{Na}\left(\mathrm{cmolc} \mathrm{dm}^{-3}\right)$ & 0,03 & 0,07 & 0,03 & 1,39 \\
\hline $\mathrm{K}\left(\mathrm{cmolc} \mathrm{dm}^{-3}\right)$ & 0,02 & 0,01 & 0,02 & 0,3 \\
\hline $\mathrm{Ca}+\mathrm{Mg}\left(\mathrm{cmolc} \mathrm{dm}^{-3}\right)$ & 0,3 & 0,2 & 1,2 & 72,7 \\
\hline $\mathrm{Al}\left(\mathrm{cmolc} \mathrm{dm}^{-3}\right)$ & 1,0 & 0,15 & 0,45 & 0,0 \\
\hline $\mathrm{H}+\mathrm{Al}\left(\mathrm{cmolc} \mathrm{dm}^{-3}\right)$ & 3,71 & 3,3 & 5,85 & 1,48 \\
\hline $\mathrm{m}(\%)$ & 74,0 & 35,0 & 26,0 & 0,0 \\
\hline $\mathrm{C}\left(\mathrm{g} \mathrm{kg}^{-1}\right)$ & 0,8 & 0,43 & 0,76 & 5,8 \\
\hline P-rem $\left(\mathrm{mg} \mathrm{L}^{-1}\right)^{(5)}$ & 22,53 & 0,49 & 0,37 & 37,32 \\
\hline CMAP $\left(\mathrm{mg} \mathrm{cm}^{-3}\right)^{(6)}$ & 0,54 & 2,13 & 2,44 & 0,31 \\
\hline $\mathrm{EA}\left(\mathrm{mg} \mathrm{L}^{-1}\right)^{-1(7)}$ & 0,92 & 0,71 & 1,11 & 0,35 \\
\hline $\mathrm{Fe}_{\mathrm{ox}}\left(\text { dag kg }^{-1}\right)^{(8)}$ & 0,28 & 0,33 & 0,38 & 0,17 \\
\hline $\mathrm{Fe}_{\mathrm{d}}\left(\mathrm{dag} \mathrm{kg}{ }^{-1}\right)^{(9)}$ & 3,55 & 4,42 & 5,83 & 0,25 \\
\hline Feox / Fed & 0,08 & 0,075 & 0,06 & 0,67 \\
\hline
\end{tabular}

${ }^{(1)} \mathrm{Ds}$ : densidade do solo; ${ }^{(2)} \mathrm{Dp}$ : densidade de partículas; ${ }^{(3)} \mathrm{Cc}$ : capacidade de campo; ${ }^{(4)} \mathrm{PMP}$ : ponto de murcha permanente; ${ }^{(5)} \mathrm{P}-\mathrm{rem}$ : fósforo remanescente; ${ }^{(7)} \mathrm{EA}$ : energia de adsorção; ${ }^{(8)} \mathrm{Fe}_{\mathrm{ox}}$ : ferro oxalato; ${ }^{(9)} \mathrm{Fe}_{\mathrm{d}}$ : ferro ditionito.

Realizaram-se correlações do FCP com as características físicas e químicas dos solos. Os dados de CMAP e EA em solos corrigidos e não corrigidos foram submetidos à análise de variância pelo teste $\mathrm{F}$, e as médias foram comparadas pelo teste de Scott-Knott $(\mathrm{p}<0,05)$.

\section{RESULTADOS E DISCUSSÃO}

Os valores do Fator intensidade (I), em unidade de potencial de fosfato dos solos $\mathrm{LAd}_{1} \mathrm{LAd}_{2}, \mathrm{NVdf}$ e VEo, foram maiores que 7,0 (Tabela 3), ou seja, compatíveis com a baixa disponibilidade natural de P dos solos (Silva et al., 2003, 2004), caracterizando uma baixa atividade de $\mathrm{P}$ em solução.
Essa baixa atividade de $\mathrm{P}$ em solução é compatível

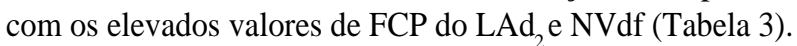
No entanto, o valor do FCP do solo LAdd é pequeno para a baixa atividade de $\mathrm{P}$ em solução (elevado I), porém, provavelmente, é compatível com o baixo fator quantidade (Q) desse solo, caracterizado pelo baixo teor de argila e elevado valor de P-rem (Tabela 2).

$\mathrm{O}$ VEo apresenta a maior atividade de $\mathrm{P}$ em solução, compatível com seu baixo FCP (Tabela 4). Pelo teor de argila desse solo (Tabela 2) mostrou-se que há potencial para elevado fator $\mathrm{Q}$, no entanto, isso não ocorre, pelo baixo FCP e alto P-rem. A mineralogia da fração argila no 


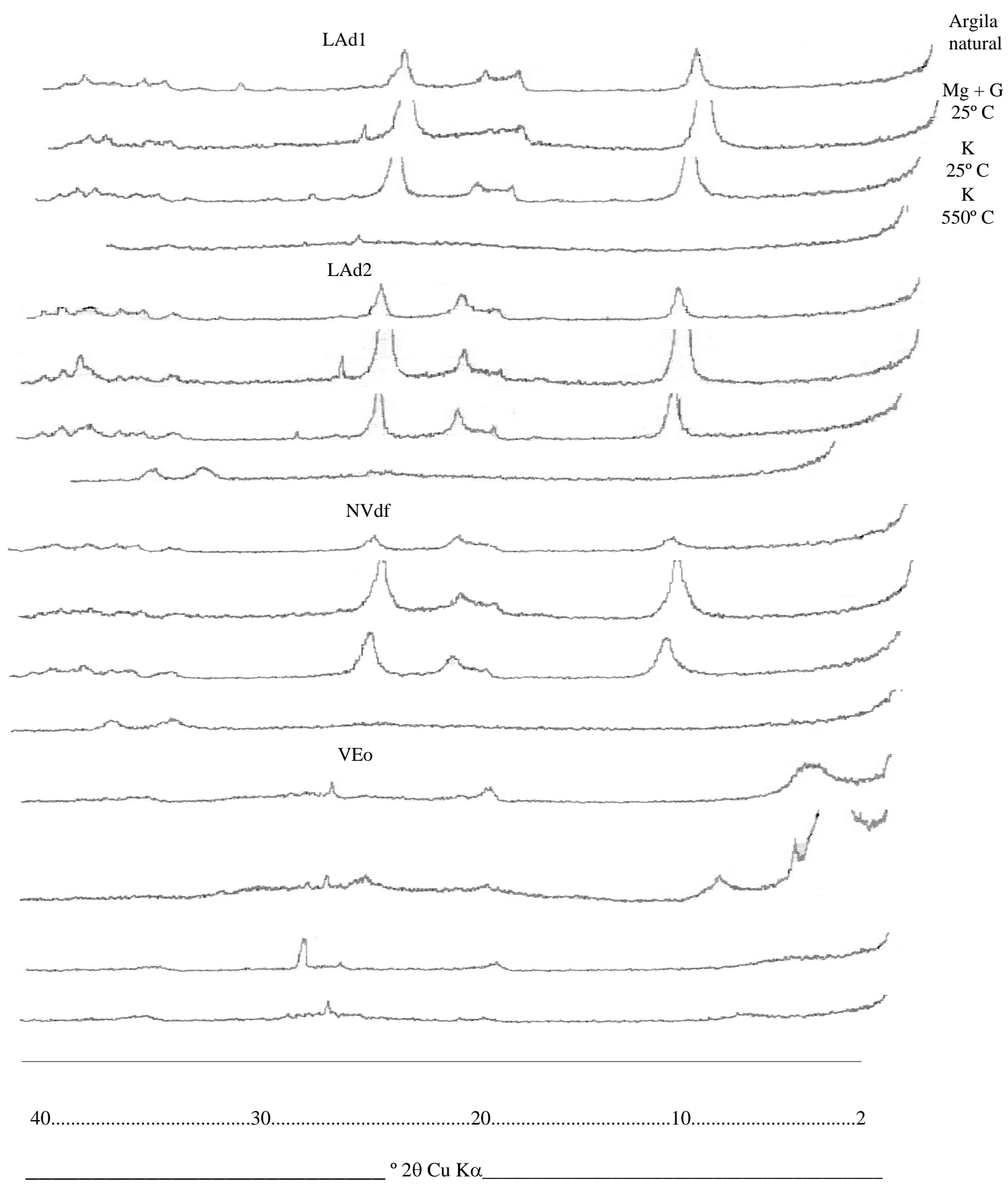

Figura 1 - Difratogramas da fração argila das amostras de solo.

Ciênc. agrotec., Lavras, v. 35, n. 1, p. 77-83, jan./fev. 2011 
VEo apresenta predominância de argilo minerais 2:1 (Figura 1), o que lhe confere uma certa semelhança com solos arenosos, quanto ao fenômeno de adsorção de P, explicando a elevada atividade de $\mathrm{P}$ em solução.

Tabela 3 - Fator intensidade e capacidade, em potencial de fosfato $\left(0,5 \mathrm{pCa}+\mathrm{pH}_{2} \mathrm{PO}_{4}\right)$, das amostras de solos.

\begin{tabular}{ccc}
\hline \multirow{2}{*}{ Solo } & Fator Intensidade (I) & Fator Capacidade (FCP) \\
\cline { 2 - 3 } & upF & $\left(10^{-8} \mathrm{~mol} / \mathrm{cm}^{3} / \mathrm{upF}\right)$ \\
\hline $\mathrm{LAd}_{1}$ & 10,91 & 65 \\
$\mathrm{LAd}_{2}$ & 9,82 & 400 \\
$\mathrm{NVdf}$ & 10,87 & 333 \\
VEo & 7,60 & 69 \\
\hline
\end{tabular}

Tabela 4 - Coeficientes de correlação linear simples entre valores do fator capacidade de fósforo (FCP) e características físicas e químicas dos solos.

\begin{tabular}{lc}
\hline Características físicas e químicas & FCP \\
\hline Argila $\left(\mathrm{dag} \mathrm{kg}^{-1}\right)$ & $0,721^{\otimes}$ \\
P-rem $\left(\mathrm{mg} \mathrm{L}^{-1}\right)^{(1)}$ & $-0,914^{*}$ \\
$\mathrm{CMAP}\left(\mathrm{mg} \mathrm{cm}^{-3}\right)^{(2)}$ & $0,893^{*}$ \\
$\left.\mathrm{EA}(\mathrm{mg} \mathrm{L})^{-1}\right)^{-1(3)}$ & $0,258^{\text {n.s }}$ \\
$\mathrm{Fe}_{\mathrm{ox}}{ }^{(4)}$ & $0,452^{\text {n.s }}$ \\
$\mathrm{Fe}_{\mathrm{d}}{ }^{(5)}$ & $0,672^{\text {n.s }}$ \\
$\mathrm{Fe}_{\mathrm{ox}} \backslash \mathrm{Fe}_{\mathrm{d}}$ & $-0,60^{\text {n.s }}$ \\
\hline
\end{tabular}

${ }^{(1)}$ Fósforo remanescente; ${ }^{(2)}$ Capacidade Máxima de Adsorção de Fósforo; ${ }^{(3)}$ Energia de adsorção; ${ }^{(4)}$ Ferro oxalato; ${ }^{(5)}$ Ferro ditionito. *; $\otimes_{\text {; n.s. }}$ significativo a $5 \%, 10 \%$ e não significativo, respectivamente.

Para enfatizar essa proposição, LAd $_{1}$ e VEo apresentaram valores de P-rem, FCP e CMAP muito próximos, enquanto o teor de argila do VEo foi, aproximadamente, três vezes superior ao do LAd $_{1}$ (Tabelas 2 e 3), indicando que a maior concentração de argila do VEo não está, de forma proporcional, influenciando no seu FCP quando comparado ao $\mathrm{LAd}_{1}$. Fica evidente que não somente a quantidade, mas também a qualidade dos constituintes minerais da fração argila do solo (Rolim Neto et al., 2004) interferem no "status" de $\mathrm{P}$ dos solos, indicado por seus valores de FCP.

As características dos solos que melhor refletiram o FCP foram o P-rem, CMAP e teor de argila (Tabela 4). Assim, como Silva et al. (2004), sugere-se a determinação do P-rem por melhor estimar o FCP dos solos e por ser de fácil determinação em laboratório de rotina.
Por ser o P-rem uma medida da CMAP de um único ponto (60 $\mathrm{mg} \mathrm{L}^{-1}$ de $\mathrm{P}$ ), em solos muito argilosos como o $\mathrm{LAd}_{2}$ e NVdf (Tabela 2), com predominância, respectivamente, de goethita e hematita na fração argila (Figura 1), essa concentração de $\mathrm{P}$ parece não ser a concentração adequada para esta análise.

$\mathrm{O}$ efeito dos constituintes mineralógicos dos solos, como hematita, goetita e caulinita, na adsorção de fosfatos, são largamente referidos em muitos trabalhos (Lopes \& Cox, 1979; Bahia Filho et al., 1982; Gomes et al., 2004; Moreira et al., 2006; Fernández et al., 2008). Por outro lado, o efeito da calagem sobre a CMAP é ainda muito discutido entre os pesquisadores (Vasconcelos et al., 1974; Parfitt, 1978; Posner \& Bowden, 1980; Haynes, 1984; Barrow, 1985). Para alguns deles (Parfitt, 1978; Haynes, 1984) adsorção de P pelo solo deve ser máxima para baixos valores de $\mathrm{pH}$, enquanto para outros (Vasconcelos et al., 1974) há pequena, mas não consistente alteração na CMAP em diferentes valores de $\mathrm{pH}$.

Neste trabalho, o $\mathrm{pH}$ final dos solos incubados situou-se entre 6,5 e 7,2. Houve pequena, porém significativa alteração da CMAP dos solos $\operatorname{LAd}_{1}$ e $\operatorname{LAd}_{2}$ (Tabela 5), ao contrário do observado por Vasconcelos et al. (1974).

A calagem de solos ricos em $\mathrm{Al}^{3+}$ como o $\mathrm{LAd}_{1} \mathrm{e}$ $\operatorname{LAd}_{2}$ (Tabela 2), tende a aumentar a adsorção de P (Viviane et al., 2010). Segundo Haynes (1984), polímeros de hidróxido de $\mathrm{Al}^{3+}$ recém formados com a calagem apresentam alta afinidade pelo P. A diminuição na CMAP do solo NVdf não foi significativa devido aos elevados teores de $\mathrm{Ca}+\mathrm{Mg}$ (Tabela 2). Segundo Novais \& Smyth (1999), o aumento da força iônica da solução de solos ácidos com a calagem e a grande presença de $\mathrm{Ca}$, mascaram o efeito esperado de maior $\mathrm{pH}$, decrescendo a adsorção de $\mathrm{P}$ dos solos. O solo VEo não foi corrigido porque o $\mathrm{pH}$ nas amostras coletadas já apresentavam valores de 6,8 (Tabela 2).

A energia de adsorção (EA) nos solos corrigidos foi, em média, significativamente menor, independentemente do solo (Tabela 5). Essa diminuição da EA pode ser explicada pelo aumento da carga superficial das partículas do solo com o aumento do $\mathrm{pH}$, aumentando a repulsão de $\mathrm{P}$ e, consequentemente, reduzindo a EA dessas partículas. Segundo alguns pesquisadores (Posner \& Bowden, 1980; Haynes, 1984; Barrow, 1985; Gonçalves et al., 1989) há uma diminuição no potencial eletrostático do plano de adsorção com o aumento do $\mathrm{pH}$ do solo. 
Tabela 5 - Capacidade máxima de adsorção de fósforo (CMAP) e energia de adsorção (EA) dos solos submetidos à correção do pH e análise da variância dos efeitos.

\begin{tabular}{|c|c|c|c|c|c|}
\hline \multirow[t]{2}{*}{ Solo } & \multicolumn{2}{|c|}{ CMAP } & \multicolumn{3}{|c|}{ EA } \\
\hline & Corrigido & Não corrigido & Corrigido & Não corrigido & Média \\
\hline $\mathrm{LAd}_{1}$ & $0,6213 \mathrm{aC}$ & $0,5906 \mathrm{bC}$ & 0,5917 & 0,8067 & $0,6992 \mathrm{C}$ \\
\hline $\mathrm{LAd}_{2}$ & $2,3246 \mathrm{aB}$ & $2,2182 \mathrm{bB}$ & 0,8389 & 0,8095 & $0,8242 \mathrm{~B}$ \\
\hline VEo & $0,3123 \mathrm{aD}$ & $0,3123 \mathrm{aD}$ & 0,3547 & 0,3547 & $0,3547 \mathrm{D}$ \\
\hline NVdf & $2,4770 \mathrm{aA}$ & $2,4850 \mathrm{aA}$ & 1,2899 & 1,3188 & $1,3043 \mathrm{~A}$ \\
\hline Média & & & $0,5188 \mathrm{~b}$ & $0,5724 \mathrm{a}$ & \\
\hline \multicolumn{6}{|c|}{$\mathrm{F}$} \\
\hline Solo & 35.8 & )*** & $987,34 * * *$ & & \\
\hline Correção & & $9 * * *$ & $3,54 *$ & & \\
\hline Solo*Correção & & $2 * * *$ & $0,94^{\mathrm{ns}}$ & & \\
\hline C.V.(\%) & & & 5,27 & & \\
\hline
\end{tabular}

Médias seguidas de mesma letra minúscula nas linhas e maiúscula nas colunas, não diferem entre si pelo teste de Scott-Knott ( $\mathrm{p}<5 \%)$. ${ }^{* * *, *, \text {, n.s }}$; significativo a $0,1 \%, 5 \%$ e não significativo, respectivamente.

\section{CONCLUSÕES}

As características que melhor refletiram o fator capacidade de $\mathrm{P}$ (FCP) foram o P remanescente (P-rem) e a capacidade máxima de adsorção de $\mathrm{P}$ (CMAP).

Independentemente dos constituintes mineralógicos da fração argila, solos com elevados teores de alumínio apresentaram aumento da CMAP com a correção.

A energia de adsorção (EA) nos solos corrigidos foi, em média, significativamente menor, independentemente do solo.

\section{REFERÊNCIAS BIBLIOGRÁFICAS}

ALVAREZ, V.H.; NOVAIS, R.F.; DIAS, L.E.; OLIVEIRA, J.A. Determinação e uso do fósforo remanescente.

Viçosa, MG: SBCS, 2000. 32p. (Boletim informativo, 25).

BAHIA FILHO, A.F.C.; VASCONCELOS, C.A.; SANTOS, H.L.; MENDES, J.F.; PITTA, G.V.E.; OLIVEIRA, A.C.

Formas de fósforo inorgânico e fósforo "disponível" em um Latossolo Vermelho-Escuro, fertilizado com diferentes fosfatos. Revista Brasileira de Ciência do Solo, Viçosa, v.6, p.99-104, 1982.

BARROW, N.J. Reaction of anions and cations with variable-charge soils. Advances in Agronomy, San Diego, v.38, p.183-230, 1985.
BRAGA, J.M.; DEFELIPO, B.V. Determinação espectrofotométrica de fósforo em extratos de solo e material vegetal. Revista Ceres, Viçosa, v.21, p.73-85, 1974.

CARNEIRO, L.F.; FURTINI NETO, A.E.; REZENDE, A.V.; CURI, N.; SANTOS, J.Z.L.; LAGO, F.J. Fontes, doses e modos de aplicação de fósforo na interação fósforo-zinco em milho. Ciência e Agrotecnologia, Lavras, v.32, p.1133-1141, 2008.

COFFIN, D.E. A method of determination of free iron in soil and clays. Canadian Journal of Soil Science, Guelph, v.43, p.7-17, 1963.

\section{EMPRESA BRASILEIRA DE PESQUISA} AGROPECUÁRIA. Manual de métodos de análise do solo. Rio de Janeiro, 1997. 212p.

FERNÁNDEZ, I.E.; NOVAIS, R.F.; NUNES, F.N.; KER, J.C. Reversibilidade de fósforo não-lábil em solos submetidos à redução microbiológica e química: II., extrações sucessivas do fósforo pela resina de troca aniônica. Revista Brasileira de Ciência do Solo, Viçosa, v.32, p.2307-2317, 2008.

FOX, R.L.; SEARLE, P.G.E. Phosphate adsorption by soils of the tropics. In: DROSDOFF, M. Diversity of soils in the tropics. Madison: American Society of Agronomy, 1978. p.97-119. 
GOMES, J.B.V.; CURI, N.; SCHULZE, D.G.; MARQUES, J.J.G.S.M.; KER, J.C.; MOTA, P.E.F. Mineralogia, morfologia e análise microscópica de solos do bioma cerrado. Revista Brasileira de Ciência do Solo, Viçosa, v.28, p.679-694, 2004.

HAYNES, R.J. Lime and phosphate in the soil-plant system. Advances in Agronomy, San Diego, v.37, p.249315, 1984.

GONÇALVES, J.L.M.; NOVAIS, R.F.; BARROS, N.F.; NEVES, J.C.L.; RIBEIRO, A.C. Cinética de transformação de fósforo-lábil em não-lábil, em solos de cerrado.

Revista Brasileira de Ciência do Solo, Viçosa, v.13, p.1324, 1989.

JACKSON, M.L. Soil chemical analysis-advanced course. Madison: University of Wisconsin, 1969. 134p.

KAMINSKI, J.; SANTOS, D.R.; GATIBONI, L.C.; MARTINS, J.R.; SANTOS, E.J.S.; TISSOT, C. Estimativa da acidez potencial em solos e sua implicação no cálculo da necessidade de calcário. Revista Brasileira de Ciência do Solo, Viçosa, v.26, p.1107-1113, 2002.

LOPES, A.S.; COX, F.R. Relação de características físicas, químicas e mineralógicas com fixação de fósforo em solos sob cerrados. Revista Brasileira de Ciência do Solo, Viçosa, v.3, p.82-88, 1979.

MESQUITA FILHO, M.V.; TORRENT, J. Phosphate sorption as related to mineralogy of a hydrosequence of soils from the Cerrado region (Brazil). Geoderma, Amnsterdam, v.58, p.107-123, 1993.

MOREIRA, F.L.M.; MOTA, F.O.B.; CLEMENTE, C.A.; AZEVEDO, B.M.; BOMFIM, G.V. Adsorção de fósforo em solos do Estado do Ceará. Revista Ciência Agronômica, Fortaleza, v.37, p.7-12, 2006.

NOVAIS, R.F.; SMYTH, T.J. Fósforo em solo e planta em condições tropicais. Viçosa, MG: UFV, 1999. 399p.

PARFITT, R.L. Anion adsorption by soils and soil materials. Advances in Agronomy, San Diego, v.30, p.150, 1978 .
POSNER, A.M.; BOWDEN, J.W. Adsorption isotherms: should they be split. Soil Science Society of America Journal, Madison, v.31, p.1-10, 1980.

ROLIM NETO, F.C.; SCHAEFER, C.E.G.R.; COSTA, L.M.; CORRÊA, M.M.; FERNANDES FILHO, E.I.; IBRAIMO, M.M. Adsorção de fósforo, superfície específica e atributos mineralógicos em solos desenvolvidos de rochas vulcânicas do Alto Paranaíba-MG. Revista Brasileira de Ciência do Solo, Viçosa, v.28, n.6, p.953964, 2004.

SCHWERTMANN, U; TAYLOR, R.M. Iron oxides. In: BIGHAM, J.M.; DIXON, J.B.; MILFORD, M.H.; ROTH, S.B.; WEED, S.B. Minerals in soil environments. Madison: Soil Sience Society of America, 1989. p.379438.

SILVA, E.M.B.; FREIRE, F.J.; SANTOS, M.V.F dos; SILVA, T.J.A. da; FREIRE, M.B.G. dos S. Níveis críticos de fósforo para Braquiaria brizantha e suas relações com características físicas e químicas em solos de Pernambuco. Revista Brasileira de Ciência do Solo, Viçosa, v.25, n.2, p.323-328, 2004.

SILVA, M.A.; NÓBREGA, J.C.A.; CURI, N.; SIQUEIRA, J.O.; MARQUES, J.J.G.S.M.; MOTTA, P.E.F. Frações de fósforo em Latossolos. Pesquisa Agropecuária Brasileira, Brasília, v.38, n.10, p.1197-1207, 2003.

TIRLONE, C.; VITORINO, A.C.T.; NOVELINO, J.O.; TIRLONE, D.; COIMBRA, D.S. Disponibilidade de fósforo em função das adições de calagem e de um bioativador no solo. Ciência e Agrotecnologia, Lavras, v.33, p.977-984, 2009.

VASCONCELOS, C.A.; BRAGA, J.M.; NOVAIS, R.F.; PINTO, O.C.B. Fósforo em dois Latossolos do Estado de Mato Grosso: Sorção de fosfato. Experientiae, Viçosa, v.18, p.267-285, 1974.

VIVIANE, C.A.; MARCHETTI, M.E.; VITORINO, AC.T.; NOVELINO, J.C.; GONÇALVES, M.C. Disponibilidade de fósforo em dois latossolos argilosos e seu acúmulo em plantas de soja, em função do aumento do pH. Ciência e Agrotecnologia, Lavras, v.34, p.61-67, 2010. 